\title{
An update on moduli stabilization with antibrane uplift
}

\author{
Emilian Dudas $^{a}$ and Severin Lüst ${ }^{a, b}$ \\ ${ }^{a}$ Centre de Physique Théorique, Ecole Polytechnique, CNRS, \\ 91128 Palaiseau Cedex, France \\ ${ }^{b}$ Institut de Physique Théorique, Université Paris Saclay, CEA, CNRS, \\ Orme des Merisiers, 91191 Gif-sur-Yvette Cedex, France \\ E-mail: emilian.dudas@polytechnique.edu, sluest@g.harvard.edu
}

ABSTRACT: It was recently shown that in warped compactifications based on a KlebanovStrassler throat there is a light complex structure field, governing the size of the throat and the redshift at its tip. We show that after uplift of the cosmological constant by an anti-D3 brane at the tip of the throat, the contribution to supersymmetry breaking coming from the new light field is large. We work out the mass scales, in particular the condition for this field to be heavier than the Kähler modulus. We check that for the range of parameters relevant for the destabilization we find agreement with de Sitter swampland conjecture. Adding matter fields on distant branes, we discuss the effects on supersymmetry breaking in the observable sector. A hierarchically small scale of supersymmetry breaking translates generically into large values of localized D3 charges in the manifold.

Keywords: Compactification and String Models, Supergravity Models, Supersymmetry Breaking, Superstring Vacua

ArXIV EPRINT: 1912.09948 


\section{Contents}

\section{Introduction 1}

2 The effective action of the Klebanov-Strassler warped compactification 2

3 Four-dimensional supergravity description and moduli stabilization $\quad 6$

$\begin{array}{lll}3.1 & \text { Vacuum structure and mass scales } & 8\end{array}$

$\begin{array}{lll}3.2 & \text { Contribution to supersymmetry breaking } & 10\end{array}$

$\begin{array}{ll}3.3 \text { Comments on the dS swampland conjecture } & 11\end{array}$

4 Adding matter: soft terms 11

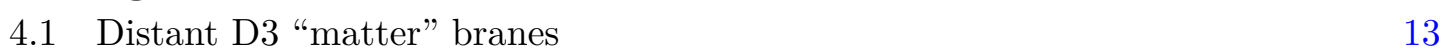

4.2 D3 branes-antibrane interactions: a higher-dimensional perspective $\quad 14$

$\begin{array}{ll}4.34 \mathrm{~d} \text { versus higher-dimensional description } & 16\end{array}$

$\begin{array}{lll}5 & \text { Conclusions } & 17\end{array}$

$\begin{array}{lr}\text { A Supergravity formulae } & 18\end{array}$

\section{Introduction}

The KKLT construction of moduli stabilization [1] relies on a three step procedure. In the first step, fluxes in the internal manifold stabilize all moduli fields, except the overall volume (Kähler) modulus $\rho .{ }^{1}$ In a second step, nonperturbative effects like stringy instantons or gaugino condensation on D7 branes stabilize $\rho$ in anti-de Sitter space. Finally, an antiD3 brane is introduced which breaks supersymmetry. ${ }^{2}$ The second and third step were debated over the years [9-21] and whereas there is not yet a consensus over the last step, there are recent positive results indicating the validity of the second step [22-29].

Recently [30, 31] the first step was addressed in the Klebanov-Strassler (KS) deformed conifold construction, which contains all ingredients needed in the KKLT construction. It was shown that one of the complex structure fields, called $S$ in what follows, which governs the size of the KS throat, is much lighter than previously thought. Its scalar potential is therefore shallow and it is significantly modified by the uplifting anti-D3 brane. Not destabilizing the throat requires a minimal value of one of the flux quanta $M$ [30-32],

$$
g_{s} M^{2} \geq(6.8)^{2} q
$$

\footnotetext{
${ }^{1}$ Here we consider only models with one Kähler modulus, i.e. $h^{1,1}=1$. In more general terms only the complex structure moduli get stabilized by fluxes.

${ }^{2}$ Actually realizing it nonlinearly, similar to perturbative string constructions of the "Brane Supersymmetry" type [2-6]), whose nonlinear supersymmetric actions were constructed in $[7,8]$.
} 
where $g_{s}$ is the string coupling and $q$ is the number of antibranes. In this paper we consider the most favorable case $q=1 .^{3}$

The purpose of the present letter is to investigate in more detail the consequences for the KKLT construction: the resulting vacuum structure and mass scales, various contributions to supersymmetry breaking and the needed localized D3 charge in the internal space which produce physically motivated hierarchies. In section 2 we review the effective action for the light complex structure field $S$ in the KS geometry and the mechanism behind the potential destabilization of the throat, once one adds the antibrane uplift. Section 3 proposes a $4 \mathrm{~d}$ supergravity description of the system including the KKLT sector of moduli stabilization and discusses the vacuum structure and supersymmetry breaking. We use a manifestly supersymmetric four-dimensional supergravity description and describe the uplift via a nilpotent chiral multiplet in supergravity [33-38]. In section 4 we add matter fields and study the effects of supersymmetry breaking, from a $4 \mathrm{~d}$ perspective and, alternatively, from a higher-dimensional one. We conclude with some comments and a short appendix.

\section{The effective action of the Klebanov-Strassler warped compactification}

The traditional KKLT construction of moduli stabilization [1] is based on warped compactifications of Calabi-Yau manifolds, with a constant dilaton, five and three-form fluxes [39]. The background metric and five-form flux are

$$
\begin{aligned}
\mathrm{d} s^{2} & =H^{-1 / 2} \mathrm{~d} s_{4}^{2}+H^{1 / 2} \mathrm{~d} s_{6}^{2}, \\
F_{5} & =(1+*) \operatorname{vol}_{4} \wedge \mathrm{d} H^{-1} \equiv * \mathcal{F}_{5}+\mathcal{F}_{5},
\end{aligned}
$$

where $H$ is the warp factor and $\mathrm{d} s_{6}^{2}$ is the unwarped metric of the internal manifold. As argued in [40], one can interpret this manifold as a throat-type region of strong warping, analogous to Randall-Sundrum type models [41, 42], glued to a compact Calabi-Yau space.

In the region of strong warping the local internal geometry is that of the deformed conifold, defined by its embedding into $\mathbb{C}^{4}, \sum_{a=1}^{4} \omega_{a}^{4}=S$. The deformation parameter $S$ is the complex structure modulus whose absolute value corresponds to the size of the 3 -sphere at the tip of the cone. The other complex structure moduli $Z^{I}$ come from the "UV" geometry. We thus have $h^{2,1}+1$ A-cycles:

$$
\int_{A} \Omega_{3}=S, \quad \int_{A_{I}} \Omega_{3}=Z^{I}
$$

where $I=0, \ldots, h^{2,1}-1$. We assume that the prepotential splits according to

$$
F\left(S, Z^{I}\right)=F_{c f}(S)+F_{U V}\left(Z^{I}\right)
$$

\footnotetext{
${ }^{3}$ It can be argued that there could be further corrections to the potential for $S$ which become relevant if one goes far away from the original, supersymmetric minimum. These corrections could potentially be so important that the bound (1.1) gets modified significantly or even becomes invalid. Resolving these issues is beyond the scope of this paper. Instead, we assume that the results of [30,31] are correct. Moreover, we will mostly work in a regime where (1.1) is satisfied and it is therefore reasonable to expect that further corrections to the potential are subleading in the region of interest.
} 
where $F_{c f}$ is the prepotential of the deformed conifold and the "UV prepotential," $F_{U V}$, does not explicitly depend on $S$. We thus have

$$
\int_{B} \Omega_{3}=F_{S}=\frac{S}{2 \pi i}\left(\log \frac{\Lambda_{0}^{3}}{S}+1\right)+F_{S}^{0}, \quad \int_{B_{I}} \Omega_{3}=F_{I},
$$

where $F_{S}$ and $F_{I}$ are the derivatives of $F$ with respect to $S$ and $Z_{I}$ respectively, and $F_{S}^{0}$ depends on the details of the compactification manifold, but is independent of $S$. The cutoff $\Lambda_{0}$ corresponds to the transition between the highly warped region, modeled as a KS throat, and (relatively unwarped) rest of the compact Calabi-Yau manifold.

The 3 -form fluxes on the 3 -cycles are ${ }^{4}$

$$
\begin{aligned}
& \frac{1}{(2 \pi)^{2} \alpha^{\prime}} F_{3}=M \alpha+M^{I} \alpha_{I}-M_{I} \beta^{I}, \\
& \frac{1}{(2 \pi)^{2} \alpha^{\prime}} H_{3}=-K \beta+K^{I} \alpha_{I}-K_{I} \beta^{I} .
\end{aligned}
$$

where $\alpha_{I}, \beta^{I}$ are Poincare duals to the cycles $B_{I}, A^{I}$ and we have singled out the RR flux on the $S^{3}$ cycle at the tip of the throat, $M$, and its NSNS partner $K$. These are the fluxes responsible for the deformation of the conifold by the parameter $S$.

The throat region is that of the Klebanov-Strassler (KS) solution [43], with the sixdimensional metric of the deformed conifold ${ }^{5}$

$$
\begin{aligned}
\mathrm{d} s_{6}^{2}=\frac{|S|^{2 / 3}}{2} \mathcal{K}(\mathcal{T})\left[\frac{1}{3 \mathcal{K}^{3}(\mathcal{T})}\left(\mathrm{d} \mathcal{T}^{2}+\left(g^{5}\right)^{2}\right)+\right. & \sinh ^{2}(\mathcal{T} / 2)\left(\left(g^{1}\right)^{2}+\left(g^{2}\right)^{2}\right) \\
& \left.+\cosh ^{2}(\mathcal{T} / 2)\left(\left(g^{3}\right)^{2}+\left(g^{4}\right)^{2}\right)\right]
\end{aligned}
$$

where $g^{i}$ is an orthogonal basis of one-forms on the base of the cone and

$$
\mathcal{K}(\mathcal{T})=\frac{(\sinh (2 \mathcal{T})-2 \mathcal{T})^{1 / 3}}{2^{1 / 3} \sinh \mathcal{T}}
$$

The warp factor of the KS solution is

$$
H(\mathcal{T})=2^{2 / 3} \frac{g_{s}\left(\alpha^{\prime} M\right)^{2}(\rho+\bar{\rho})}{|S|^{4 / 3}} I(\mathcal{T})
$$

where

$$
I(\mathcal{T})=\int_{\mathcal{T}}^{\infty} \mathrm{d} x \frac{x \operatorname{coth} x-1}{\sinh ^{2} x}(\sinh (2 x)-2 x)^{1 / 3}
$$

The UV cutoff $\Lambda_{0}$ where the solution is glued to the compact Calabi-Yau solution is such that the total NSNS flux over the $B$ cycle is $K$, according to (2.5):

$$
K=\frac{1}{(2 \pi)^{2} \alpha^{\prime}} \int_{B} H_{3}=\frac{1}{(2 \pi)^{2} \alpha^{\prime}} \int_{\mathcal{T} \leq \mathcal{T}_{0}} \int_{S^{2}} H_{3}, \quad \Lambda_{0}^{2}=\frac{3}{2^{5 / 3}}|S|^{2 / 3} e^{2 \mathcal{T}_{0} / 3} .
$$

\footnotetext{
${ }^{4}$ The setup only requires one type of flux on each cycle.

${ }^{5}$ Note that taking $\mathcal{T}$ and $g^{i}$ to be dimensionless requires the deformation parameter $S$ to be of dimension $(\text { length })^{3}$.
} 
On a compact manifold the Bianchi identity for the five-form flux leads to the tadpole cancelation condition forcing the total D3-charge of the solution to be zero.

$$
M K+M^{I} K_{I}-M_{I} K^{I}+Q_{3}^{\mathrm{loc}}=0,
$$

where the charge of localized D3-brane and O3-plane sources is ${ }^{6}$

$$
Q_{3}^{\text {loc }}=N_{\mathrm{D} 3}-\frac{1}{4} N_{O 3} .
$$

The tadpole condition (2.11) leads to a upper bound on the product of fluxes allowing the cancelation of $C_{4}$ flux

$$
M K \leq\left|Q_{3}^{\mathrm{loc}}\right| .
$$

Recently it was shown [30, 31] that in the strongly warped region of the KlebanovStrassler compactification, the light complex structure field $S$ can be destabilized by the $\overline{\mathrm{D} 3}$ uplift. The potential for the complex structure modulus $S$ involves the fluxes $M$ and $K$, while it depends on the other fluxes only indirectly through the axion-dilaton $\tau$, whose vev is determined by all fluxes. Furthermore, unlike the other "bulk" moduli, the potential for $S$ is highly affected by the warp factor. Its functional form, in the Einstein frame, derived in $[44,45]$ is

$$
\begin{aligned}
V_{\mathrm{KS}} & =\frac{\pi^{3 / 2}}{\kappa_{10}} \frac{g_{s}}{(\rho+\bar{\rho})^{3}}\left[c \log \frac{\Lambda_{0}^{3}}{|S|}+c^{\prime} \frac{g_{s}\left(\alpha^{\prime} M\right)^{2}}{|S|^{4 / 3}}\right]^{-1}\left|\frac{M}{2 \pi i} \log \frac{\Lambda_{0}^{3}}{S}+i \frac{K}{g_{s}}\right|^{2} \\
& \simeq \frac{\pi^{3 / 2}|S|^{4 / 3}}{\kappa_{10} c^{\prime}\left(\alpha^{\prime} M\right)^{2}(\rho+\bar{\rho})^{3}}\left|\frac{M}{2 \pi i} \log \frac{\Lambda_{0}^{3}}{S}+i \frac{K}{g_{s}}\right|^{2}
\end{aligned}
$$

where in the last line we used the approximation of strong warping. Moreover, $g_{s}$ is the stabilized vev of the dilaton, $\rho+\bar{\rho}=\left(\mathrm{Vol}_{6}\right)^{3 / 2}, c$ denotes the constant value of the warp factor at the UV and will not be relevant here, whereas the constant $c^{\prime}$, multiplying the term coming solely from the warp factor, denotes an order one coefficient, whose approximate numerical value was determined in [44] to be $c^{\prime} \approx 1.18$. The potential (2.14) has a supersymmetric minimum

$$
S_{\mathrm{KS}}=\Lambda_{0}^{3} e^{-\frac{2 \pi K}{g_{s} M}}
$$

which is exponentially small for appropriate values of the fluxes $(M, K)$. Since the field $S$ has mass dimension -3 , whereas the corresponding gauge theory condensate $Z$ has dimension 3 , one can write the potential in terms of $Z$ in the following way. Writing the $10 \mathrm{~d}$ metric in the form

$$
d s^{2}=e^{2 A} d s_{4}^{2}+e^{-2 A} t^{1 / 2} d s_{6}^{2},
$$

where the volume of the internal space is parametrized in terms of $t=\rho+\bar{\rho}$. The relation between the $10 \mathrm{~d}$ and the $4 \mathrm{~d}$ Newton constant is

$$
\frac{1}{\kappa_{4}^{2}}=\frac{V_{w}}{\kappa_{10}^{2}}, \quad \text { where } \quad V_{w}=\int d^{6} y \sqrt{g_{6}} e^{-4 A},
$$

\footnotetext{
${ }^{6}$ There can be also an additional contribution to $Q_{3}^{\text {loc }}$ coming from D7-branes and O7-branes.
} 
where $V_{w}$ is a fiducial volume. Using the relation $2 \kappa_{10}^{2}=(2 \pi)^{7} \alpha^{4}$ and redefining the $S$ field according to

$$
S=\left(2^{3 / 4} \pi^{1 / 2} \alpha^{\prime}\right)^{3} Z,
$$

one arrives at the $4 \mathrm{~d}$ scalar potential in the Einstein frame ${ }^{7}$

$$
V_{\mathrm{KS}}=\frac{|Z|^{4 / 3}}{c^{\prime} M^{2}(\rho+\bar{\rho})^{2}}\left|\frac{M}{2 \pi i} \log \frac{\Lambda^{3}}{Z}+i \frac{K}{g_{s}}\right|^{2},
$$

where we have redefined $\Lambda_{0} \rightarrow \Lambda$ analogously to (2.18) and from now on one sets $\kappa_{4}=1$. The scale $\Lambda$ in (2.19) has now mass dimension one. Later on one will define a more canonical dimension-one field $Z \sim Y^{3}$.

An anti-D3 brane at the tip of the throat uplifts the KS potential (2.19). The contribution to the potential is determined from

$$
S_{\mathrm{D} 3}=S_{\mathrm{DBI}}+S_{\mathrm{CS}}=-T_{3} \int \mathrm{d}^{4} x \sqrt{-g_{4}}\left[1+\mathcal{O}\left(\alpha^{\prime 2}\right)\right] \pm T_{3} \int C_{4},
$$

where the sign in front of the second term is determined by the charge of the brane, and $T_{3}$ is given by $T_{3}=\frac{1}{(2 \pi)^{3} \alpha^{\prime 2}}$. For the D3-brane in a background given by (2.1), the DBI and the CS pieces of the action cancel each other. Hence, for the $\overline{\mathrm{D} 3}$-brane they add up and one finds

$$
V_{\overline{\mathrm{D} 3}}=-2 T_{3} C_{4}=\frac{2}{(2 \pi)^{3} \alpha^{\prime 2}} H^{-1} .
$$

Using the warp factor given in (2.8) and turning into the 4d Einstein frame, one finally obtains

$$
V_{\overline{\mathrm{D} 3}}=\frac{1}{\pi(\rho+\bar{\rho})^{2}} \frac{2^{1 / 3}}{I(\mathcal{T})} \frac{|Z|^{4 / 3}}{g_{s} M^{2}} .
$$

The $I(\mathcal{T})$, defined in $(2.9)$ is a monotonically decreasing function. Therefore, a $\overline{\mathrm{D} 3}$-brane has minimal energy if it is placed at the tip of the throat. For later convenience we introduce a constant $c^{\prime \prime}=\frac{2^{1 / 3}}{I(0)} \approx 1.75$.

With these notations and in the highly warped region, the total potential takes the form

$$
V_{\mathrm{KS}+\text { uplift }}=\frac{|Z|^{4 / 3}}{(\rho+\bar{\rho})^{2} c^{\prime} M^{2}}\left\{\left|\frac{M}{2 \pi i} \log \frac{\Lambda^{3}}{Z}+i \frac{K}{g_{s}}\right|^{2}+\frac{c^{\prime} c^{\prime \prime}}{\pi g_{s}}\right\} .
$$

The minimum of the potential with the uplift can be found analytically to be given by [30]

$$
Z_{0}=e^{-\frac{3}{4}\left(1-\sqrt{1-\frac{64 \pi c^{\prime} c^{\prime \prime}}{9 g_{s} M^{2}}}\right)} \Lambda^{3} e^{-\frac{2 \pi K}{g_{s} M}}
$$

which clearly displays the disappearence of the non-trivial minimum for small values of the fluxes $g_{s} M^{2}<\left(64 \pi c^{\prime} c^{\prime \prime}\right) / 9$, leading to the condition (1.1).

\footnotetext{
${ }^{7}$ We take also into account the change of power $1 /(\rho+\bar{\rho})^{3} \rightarrow 1 /(\rho+\bar{\rho})^{2}$ due to the warping, as argued for in [46].
} 
Some caution is however required in using the scalar potential $(2.23) .{ }^{8}$ In the KS solution the $S$ field is not a modulus, but is fixed to its supersymmetric value (2.15). Replacing the warp factor with its $S$-dependence as in (2.22) is well justified only at the minimum and might not be trusted far from it. So the potential (2.23) might not be really trusted "off-shell" far from its KS minimum. For large values of $M$-flux, the shift in the vev induced by the antibrane uplift is small and the potential should be reliable close to the new minimum. For smaller values of the flux, below to the destabilization point (1.1), the runaway behavior depends crucially on the form of the potential for small values of $S$, far away from the old supersymmetric minimum. Therefore, it cannot be excluded that there are significant corrections to the potential which modify or invalidate the bound (1.1). On the other hand, the results of [32] provide further evidence for the validity of (1.1) and thus also of the potential (2.23). There, a similar bound was obtained from the numerical construction of Klebanov-Strassler black holes [47] which goes beyond the probe approximation and takes the full back reaction on the geometry into account. Moreover, [83] compared the analysis of $[30,31]$ with the radion stabilization in RS scenarios by the Goldberger-Wise formalism and found qualitative agreement.

In this paper we will no try to resolve these issues and will take the validity of the potential (2.23) as a working assumption. Moreover, we mostly work in the regime of large enough $g_{s} M^{2}$ where the shift in $S$ induced by the $\overline{\mathrm{D} 3}$ brane is small. It is therefore reasonable to assume that in the vicinity of the $\overline{\mathrm{D} 3}$ minimum possible corrections to the potential are subleading. Notice, that even in this regime of parameters the effects of the warp factor on the potential $[44,45]$ are crucial for our following analysis. Neglecting them corresponds to setting $c^{\prime}=0$ and results in a significantly different mass spectrum.

\section{Four-dimensional supergravity description and moduli stabilization}

Adding the nonperturbative term generated by stringy instantons or gaugino condensation amounts to adding to the perturbative superpotential the KKLT-like terms $W_{\mathrm{KKLT}}=$ $W_{0}+A e^{-a \rho}$. The KKLT sector by itself will be defined by

$$
\mathcal{K}=-3 \log (\rho+\bar{\rho}), \quad W_{\mathrm{KKLT}}=W_{0}+A e^{-a \rho},
$$

which leads to the KKLT potential

$$
V_{\mathrm{KKLT}}=\frac{1}{(\rho+\bar{\rho})^{3}}\left\{\frac{(\rho+\bar{\rho})^{2}}{3}\left|\partial_{\rho} W_{\mathrm{KKLT}}-\frac{3}{\rho+\bar{\rho}} W_{\mathrm{KKLT}}\right|^{2}-3\left|W_{\mathrm{KKLT}}\right|^{2}\right\} \text {, }
$$

which has an AdS minimum. We will show that, under some mild assumptions, the KKLT sector does not affect the conifold destabilization mechanism we found. Before showing this, we remind the reader that the uplift can also be described in a manifestly supersymmetric formalism using nonlinear supersymmetry with a nilpotent goldstino superfield [33-38, 4852]. Therefore, if there is a mass gap we should be able to describe the whole action in

\footnotetext{
${ }^{8}$ We thank A. Hebecker and L. Martucci for useful discussions on this point.
} 
terms of a supergravity action. Introducing a nilpotent superfield $X$, it is indeed possible to write the Kähler potential at the perturbative level as

$$
\begin{aligned}
\mathcal{K} & =-3 \log \left(\rho+\bar{\rho}-\frac{|X|^{2}}{3}-\frac{\xi|Z|^{2 / 3}}{3}\right)-\log (-i(\tau-\bar{\tau}))+c|Z|^{2}\left(\log \frac{\Lambda^{3}}{|Z|}+1\right), \\
W & =W_{0}+A e^{-a \rho}+\frac{M}{2 \pi i} Z\left(\log \frac{\Lambda^{3}}{Z}+1\right)+K \tau Z+\frac{1}{M} \sqrt{\frac{c^{\prime \prime}}{\pi}} Z^{2 / 3} \tau X
\end{aligned}
$$

where we introduced the (flux-dependent) constant $\xi=9 c^{\prime} g_{s} M^{2} . W_{0}$ denotes the vev of the bulk or "UV" superpotential. In what follows, we assume the dilaton to be stabilized $\tau=\frac{i}{g_{s}}$. The field $X$ satisfies the nilpotent constraint $X^{2}=0$. It contains the goldstino $G$ localized on the antibrane and generates the Volkov-Akulov nonlinear supersymmetric Lagrangian. The solution of the constraint, in superspace language is

$$
X=\frac{G G}{2 F_{X}}+\sqrt{2} \theta G+F_{X} \theta^{2},
$$

where $\theta$ is the fermionic superspace coordinate. The simplest recent string theory examples of Volkov-Akulov nonlinear supersymmetric actions consists of putting a stuck $\overline{\mathrm{D} 3}$ antibrane on top of an $\mathrm{O}_{\text {- }}$ plane [53], which reduces the (anti)brane localized degrees of freedom to only the goldstino [48-52]. Similar construction, much like the original string vacua with "brane supersymmetry breaking" [2-6] also generate a nonlinear realization of supersymmetry on the antibranes, as shown explicitly in $[7,8]$. The nilpotent constraint eliminates the scalar partner of the goldstino, keeping the auxiliary field $F_{X}$. Consequently, the scalar potential is computed from the usual supergravity potential, by setting at the end $X=0$. The last term in the superpotential reproduces the antibrane uplift, redshifted by the S-dependent prefactor. Note that the nilpotent goldstino formalism is valid as long as $F_{X} \neq 0$. In the example we consider (3.5), we find ${ }^{9}$ that $F_{X}=\frac{i}{g_{s} M} \sqrt{\frac{c^{\prime \prime}}{\pi}} Z^{2 / 3}$ and, since $\langle Z\rangle \neq 0$, the formalism is indeed valid. The stronger the warping the smaller the supersymmetry breaking. We expect in principle a maximum value of the warping also from the requirement that states decoupled by the supersymmetry breaking to be heavy enough.

This Kähler potential should be understood as an $Z$-expansion of the general Kähler potential derived in [54] (see also [55-57]), reproducing the metric $G_{S \bar{S}}$ of $[44,45]$.

A naive integration-out of $Z$ would produce an effective constant

$$
W_{0, \text { eff }}=W_{0}+\frac{M}{2 \pi i}\left(1+\sqrt{1-\frac{64 \pi c^{\prime} c^{\prime \prime}}{9 g_{s} M^{2}}}\right) Z_{0} .
$$

It is convenient in what follows to work with a dimension 1-field $Y$ instead of the dimension 3 one $Z$. We introduce the convenient definitions

$$
\begin{aligned}
Y & =\left(\frac{\xi}{3}\right)^{1 / 2} Z^{1 / 3}=\left(\frac{3 c^{\prime} g_{s} M^{2}}{2^{3 / 2} \pi \alpha^{\prime 2}}\right)^{1 / 2} S^{1 / 3} \\
\tilde{M} & =\left(\frac{3}{\xi}\right)^{3 / 2} M, \quad \tilde{K}=\left(\frac{3}{\xi}\right)^{3 / 2} K, \quad \tilde{\Lambda}_{0}=\left(\frac{\xi}{3}\right)^{1 / 2} \Lambda, \quad \tilde{c}^{\prime \prime}=\frac{3}{\xi g_{s} M} \sqrt{\frac{c^{\prime \prime}}{\pi}} .
\end{aligned}
$$

\footnotetext{
${ }^{9}$ The factor i can be removed by a redefinition of the field $X$.
} 
With these definitions, freezing the dilaton and in the region of strong warping, where the perturbative Kähler potential for S is negligible, the effective action (3.3) becomes

$$
\begin{aligned}
\mathcal{K} & =-3 \log \left(\rho+\bar{\rho}-\frac{|X|^{2}}{3}-|Y|^{2}\right), \\
W & =W_{0}+A e^{-a \rho}+\frac{\tilde{M}}{2 \pi i} Y^{3}\left(3 \log \frac{\tilde{\Lambda}_{0}}{Y}+1\right)+\frac{i \tilde{K}}{g_{s}} Y^{3}+\tilde{c}^{\prime \prime} Y^{2} X .
\end{aligned}
$$

Therefore, the SUGRA scalar potential of the model (3.7) can be written in the form

$$
V=\frac{1}{r^{2}}\left\{3|Y|^{4}\left|\frac{3 \tilde{M}}{2 \pi i} \log \frac{\tilde{\Lambda}_{0}}{Y}+i \frac{\tilde{K}}{g_{s}}\right|^{2}+\left|\tilde{c^{\prime \prime}} Y^{2}\right|^{2}+\left|\partial_{\rho} W-\frac{3}{\rho+\bar{\rho}} W_{\mathrm{eff}}\right|^{2}-\frac{3}{\rho+\bar{\rho}}\left|W_{\mathrm{eff}}\right|^{2}\right\}
$$

where we defined

$$
W_{\text {eff }}=W_{0}+A e^{-a \rho}+\frac{\tilde{M}}{2 \pi i} Y^{3} \quad \text { and } \quad r=\rho+\bar{\rho}-|Y|^{2} .
$$

The first two terms in (3.8) recover the potential $V_{\mathrm{KS}+\text { uplift }}$ displayed in (2.23) coming from the fluxes and the $\overline{\mathrm{D} 3}$ uplift, written in terms of the dimension-one field $Y$ and in the small $Y$ limit. The last two terms in the potential (3.8) reduce, for $Y=0$, to the KKLT potential for $\rho$.

\subsection{Vacuum structure and mass scales}

The scalar potential (3.8) has an almost decoupled structure, between the KS and the KKLT sector. In the case that one of the moduli is significantly heavier than the other, this implies that the decoupled KS+uplift and the KKLT+uplift minima will be a good zeroth order approximation. The later ones are determined as

$$
\left.\frac{\partial V_{\mathrm{KS}+\text { uplift }}}{\partial Y}\right|_{Y_{0}}=0,\left.\quad \frac{\partial V_{\mathrm{KKLT}+\text { uplift }}}{\partial \rho}\right|_{\rho_{0}}=0 .
$$

The explicit values are

$$
\begin{aligned}
& Y_{0}=e^{-\frac{2 \pi \epsilon_{0}}{3 \tilde{M}}} \tilde{\Lambda}_{0} e^{-\frac{2 \pi \tilde{K}}{3 g_{s} \tilde{M}}} \leftrightarrow \quad Z_{0}=e^{-\frac{3}{4}\left(1-\sqrt{1-\frac{64 \pi c^{\prime} c^{\prime \prime}}{9 g_{s} M^{2}}}\right)} \Lambda_{0}^{3} e^{-\frac{2 \pi K}{g_{s} M}} \\
& {\left[a\left(\rho_{0}+\bar{\rho}_{0}\right)+5\right] A e^{-a \rho_{0}}=-3 W_{0}}
\end{aligned}
$$

The constant $\epsilon_{0}$ above is given by

$$
\begin{aligned}
\epsilon_{0} & =\frac{3 \tilde{M}}{2 \pi} \log \frac{\tilde{\Lambda}_{0}}{Y_{0}}-\frac{\tilde{K}}{g_{s}}=\frac{1}{4}\left(\frac{3 \tilde{M}}{2 \pi}-\sqrt{\left(\frac{3 \tilde{M}}{2 \pi}\right)^{2}-\frac{48 \tilde{c}^{\prime \prime 2}}{9}}\right) \\
& =\frac{3}{8 \pi M^{2}\left(3 c^{\prime} g_{s}\right)^{3 / 2}}\left(1-\sqrt{1-\frac{64 \pi c^{\prime} c^{\prime \prime}}{9 g_{s} M^{2}}}\right),
\end{aligned}
$$


and measures the deviation of the minimum of $S_{0}$ from the supersymmetric one (2.15) before the uplift. Notice that the higher the flux $M$ the smaller the deviation of the vev of $Z$ from its supersymmetric value. The main result of [30] was that the existence of a non-trivial minimum for $S$ implies a minimum value for the $M$ flux

$$
g_{s} M^{2} \geq M_{\min }^{2}=\frac{64 \pi c^{\prime} c^{\prime \prime}}{9} \simeq(6.8)^{2} .
$$

The cancelation of the cosmological constant after uplift translates into the tuning

$$
\left(\left|\tilde{c}^{\prime \prime}\right|^{2}+3 \epsilon_{0}^{2}\right)\left|Y_{0}\right|^{4} \simeq \frac{3\left|W_{0}\right|^{2}}{\rho_{0}+\bar{\rho}_{0}} \quad \rightarrow \quad W_{0} \simeq \epsilon_{0}\left(\rho_{0}+\bar{\rho}_{0}\right)^{1 / 2} Y_{0}^{2},
$$

where in the last estimate we neglected $|\tilde{c}| \ll \epsilon_{0}$, valid for not extremely large values of the flux. Notice that an uplift to zero cosmological constant requires $W_{0} \sim Z_{0}^{2 / 3}$ which is much larger (in the strong warping regime) than the last term in (3.5), induced by integrating-out the field $Z$. The mass of the moduli fields are then readily computed. We denote by $m_{Y, \pm}$ the two mass eigenstates of the complex field $Y$. At the leading order one finds

$$
\begin{array}{rlrl}
m_{Y,+}^{2} & =\frac{\tilde{M}}{4 \pi} \frac{Y_{0}^{2}}{\rho_{0}+\bar{\rho}_{0}}=\frac{Z_{0}^{2 / 3}}{8 \pi \rho_{0}\left(3 c^{\prime} g_{s}\right)^{1 / 2}}, & m_{Y,-}^{2}=m_{Y,+}^{2} \sqrt{1-\frac{M_{\min }^{2}}{g_{s} M^{2}}}, \\
m_{\rho \bar{\rho}}^{2}=\frac{a^{2} W_{0}^{2}}{\rho_{0}+\bar{\rho}_{0}}, & m_{\rho \rho}^{2} \ll m_{\rho \bar{\rho}}^{2} .
\end{array}
$$

After imposing the cancelation of the vacuum energy, the ratio of the moduli masses is given by

$$
\frac{m_{\rho}^{2}}{m_{Y}^{2}} \sim\left(1-\sqrt{1-\frac{M_{\min }^{2}}{g_{s} M^{2}}}\right)^{2} \frac{a^{2}\left(3 c^{\prime}\right)^{5 / 2} \rho_{0} Z_{0}^{2 / 3}}{32 \pi^{2} g_{s}^{1 / 2}} .
$$

The $Z$ modulus is therefore heavier than $\rho$ provided that

$$
Z_{0} \ll\left[\frac{32 \pi^{2} g_{s}^{1 / 2}}{\left(1-\sqrt{1-\frac{M_{\min }^{2}}{g_{s} M^{2}}}\right)^{2}\left(3 c^{\prime}\right)^{5 / 2}}\right]^{\frac{3}{2}} \frac{1}{\left(a^{2} \rho_{0}\right)^{3 / 2}} .
$$

This is also the condition of validity for the (quasi) decoupling of the KS and KKLT sectors, that was our starting assumption in finding the vacuum structure. One can aposteriori check that the shifts in the minima after coupling the KS+uplift and the KKLT sector are parametrically $\delta Y / Y_{0} \sim \delta \rho / \rho_{0} \sim Y_{0} \sqrt{\rho_{0}}$, which are small precisely when (3.17) is fulfilled. The constraint (3.17) is easy to satisfy for large fluxes. The strongest constraint arises for small fluxes. For example, close to the critical value (3.13) and in the case of stringy instantons $a=2 \pi$, (3.17) gives roughly $Z_{0} \ll g_{s}^{3 / 4} /\left(3 \rho_{0}\right)^{3 / 2}$. Since

$$
\frac{2 \pi K}{g_{s} M}=\frac{2 \pi M K}{g_{s} M^{2}} \leq \frac{2 \pi\left|Q_{3}^{\mathrm{loc}}\right|}{(6.8)^{2}}
$$


a given value of the warp factor translates into a minimum value of the localized D3 charge $Q_{3}^{\text {loc }}$, according to

$$
\left|Q_{3}^{\text {loc }}\right| \geq \frac{(6.8)^{2}}{2 \pi}\left|\log Z_{0}\right|
$$

For example, typical KKLT values $\rho_{0} \sim 20-50$ and for $\Lambda_{0} \sim M_{P}$ imply $Z_{0}<10^{-2}$ to satisfy (3.17), which would imply $\left|Q_{3}^{\text {loc }}\right| \geq 40-50$. As another example, the gravitino mass is given by

$$
m_{3 / 2} \simeq \frac{W_{0}}{\left(\rho_{0}+\bar{\rho}_{0}\right)^{3 / 2}} \sim \frac{\epsilon_{0} Y_{0}^{2}}{\rho_{0}},
$$

where the last estimate is order of magnitude only. $\mathrm{TeV}$ values of the gravitino mass $m_{3 / 2} \sim \mathrm{TeV}$ would require $Z_{0} \sim 10^{-21}$, which translates into $\left|Q_{3}^{\text {loc }}\right| \geq 350-400$. Whereas this is possible in F-theory, it is challenging to construct explicit examples with such large localized D3 charges [58].

\subsection{Contribution to supersymmetry breaking}

The contribution of various fields to supersymmetry breaking is encoded in the auxiliary fields, in terms of which one can write the scalar potential as

$$
V=\mathcal{K}_{I \bar{J}} F^{I} F^{\bar{J}}-3 m_{3 / 2}^{2}, \quad \text { where } \quad F^{I}=e^{\mathcal{K} / 2} \mathcal{K}^{I \bar{J}} \overline{D_{J} W} .
$$

Using the vev's obtained in the previous section, one finds

$$
D_{X} W=\frac{1}{g_{s} M} \sqrt{\frac{c^{\prime \prime}}{\pi}} Z_{0}^{2 / 3}, \quad D_{Y} W \simeq-3 i \epsilon_{0} Y_{0}^{2}, \quad D_{\rho} W \simeq-\frac{6 W_{0}}{a\left(\rho_{0}+\bar{\rho}_{0}\right)^{2}} .
$$

Even if the fields $Y$ and $\rho$ mix in the Kähler potential, it can be shown that it is a good approximation to neglect the mixing and to define individual contributions to SUSY breaking, according to

$$
f_{I} \equiv e^{\mathcal{K} / 2}\left(\mathcal{K}^{I \bar{I}}\right)^{1 / 2} \overline{D_{I} W}, \quad V \simeq \sum_{I}\left|f_{I}\right|^{2}-3 m_{3 / 2}^{2}
$$

Then (3.22) leads to

$$
f_{X} \sim f_{Y} \sim m_{3 / 2}, \quad f_{\rho} \sim \frac{m_{3 / 2}}{a\left(\rho_{0}+\bar{\rho}_{0}\right)}
$$

It is interesting that the conifold field $Y \sim Z^{1 / 3}$ has a large contribution to supersymmetry breaking, at the same order as the one of the uplift (nilpotent) field $X$. Both contributions are localized at the tip of the throat. Notice that a contribution to supersymmetry breaking of the complex structure field $Z$ could be interpreted as an effective generation of an $(1,2)$ flux. On the other hand, the contribution to supersymmetry breaking of the Kähler modulus $\rho$, which propagates across the whole bulk, is suppressed by a factor of order $1 /\left(a \rho_{0}\right)$. This will have consequences on the transmission of supersymmetry breaking into the matter sector. 


\subsection{Comments on the dS swampland conjecture}

It was recently conjectured [59-61] that in all controlled compactifications

$$
|\nabla V| \geq a V \quad \text { or } \quad \min \left(\nabla_{i} \nabla_{j} V\right) \leq-a^{\prime} V
$$

where $a, a^{\prime}>0$ are $\mathcal{O}(1)$ numbers. The second condition is not constraining in our case, therefore we discuss the first one. Since the KS and the KKLT sector are approximately decoupled in the regime $m_{\rho} \ll m_{Y}$, we can concentrate on the KS plus the uplift sector, assuming the Kähler modulus $\rho$ is stabilized, described by

$$
\mathcal{L}_{Y}=\frac{3}{\rho+\bar{\rho}}|\partial Y|^{2}-V_{\mathrm{KS}+\text { uplift }},
$$

where the appropriate scalar potential is given in (2.23). We therefore compute

$$
\frac{\left|\nabla_{Y} V\right|}{V}=\frac{\sqrt{G^{Y \bar{Y}} \partial_{Y} V \partial_{\bar{Y}} V}}{V} .
$$

If $\sqrt{g_{s}} M \geq M_{\min }$, there is a dS minimum and the dS conjecture is violated. If this is realized in string theory depends on the existence of compactifications with large localized D3 charges [58]. Following the same steps and arguments as in [30], another check can be performed for small flux $\sqrt{g_{s}} M<M_{\min }$, where the dS minimum disappear. One finds

$$
\frac{\left|\nabla_{Y} V\right|}{V} \geq \frac{2}{|Y|} \sqrt{\frac{\rho+\bar{\rho}}{3}}\left(1-\frac{\sqrt{g_{s}} M}{M_{\min }}\right) .
$$

This is generically satisfied in the limit of strong warping. It is however amusing to notice that by imposing $a \sim 1$ one obtains a condition parametrically of the type (3.17), although the two conditions apply to different cases.

On the other hand, a sufficient (but not necessary) condition to satisfy the dS conjecture would be to select large enough values of the $(0,3)$ flux parameter $W_{0}$ to forbid an uplift to positive vacuum energy. Using the results and notations from section 3.1, this condition reads

$$
W_{0} \geq \epsilon_{0}\left(\rho_{0}+\bar{\rho}_{0}\right)^{1 / 2} Y_{0}^{2} .
$$

\section{Adding matter: soft terms}

The Klebanov-Strassler throat can generate an exponential hierarchy for the scale of supersymmetry breaking in the observable sector. The matter fields $Q_{i}$ are defined as usual by the properties $\left\langle Q_{i}\right\rangle=0, F^{i}=0$. The $4 \mathrm{~d}$ supergravity lagrangian contains hidden sector (moduli) fields called $T_{\alpha}$ in what follows $\left(T_{\alpha}=X, Y, \rho\right.$ in our case), coupled to the matter fields $Q_{i}$. The Kähler potential and superpotential are defined by an expansion in powers of the matter fields

$$
\begin{aligned}
\mathcal{K} & =\hat{\mathcal{K}}\left(T_{\alpha}, \bar{T}_{\alpha}\right)+K_{i \bar{\jmath}}\left(T_{\alpha}, \bar{T}_{\alpha}\right) Q^{i} \bar{Q}^{\bar{\jmath}}+\frac{1}{2}\left(Z_{i j}\left(T_{\alpha}, \bar{T}_{\alpha}\right) Q^{i} Q^{j}+\text { h.c. }\right), \\
W & =\hat{W}\left(T_{\alpha}\right)+\frac{1}{2} \tilde{\mu}_{i j}\left(T_{\alpha}\right) Q^{i} Q^{j}+\frac{1}{3} \tilde{Y}_{i j k}\left(T_{\alpha}\right) Q^{i} Q^{j} Q^{k}+\cdots
\end{aligned}
$$


The low-energy softly broken supersymmetric lagrangian is defined by the superpotential and soft scalar potential

$$
\begin{aligned}
& W_{\text {eff }}=\frac{1}{2} \mu_{i j} Q^{i} Q^{j}+\frac{1}{3} Y_{i j k} Q^{i} Q^{j} Q^{k} \\
& \mathcal{L}_{\text {soft }}=-m_{i \bar{\jmath}}^{2} q^{i} q^{\bar{\jmath}}-\left(\frac{1}{2} B_{i j} q^{i} q^{j}+\frac{1}{3} A_{i j k} q^{i} q^{j} q^{k}+\frac{1}{2} M_{a} \lambda_{a} \lambda_{a}+\text { h.c. }\right),
\end{aligned}
$$

where $Y_{i j k}=e^{K / 2} \tilde{Y}_{i j k}$. For zero cosmological constant, the general tree-level expressions for soft terms and supersymmetric $\mu$-terms in $4 \mathrm{~d}$ supergravity, are given by [62-66]

$$
\begin{aligned}
m_{i \bar{\jmath}}^{2}= & m_{3 / 2}^{2}\left(G_{i \bar{\jmath}}-G^{\alpha} G^{\bar{\beta}} R_{i \bar{\jmath} \alpha \bar{\beta}}\right)=m_{3 / 2}^{2} K_{i \bar{\jmath}}-F^{\alpha} F^{\bar{\beta}} R_{i \bar{\jmath} \alpha \bar{\beta}}, \\
\mu_{i j}= & m_{3 / 2} \nabla_{i} G_{j}=e^{\frac{K}{2}} \tilde{\mu}_{i j}+m_{3 / 2} Z_{i j}-F^{\bar{\alpha}} \partial_{\bar{\alpha}} Z_{i j}, \\
A_{i j k}= & m_{3 / 2}^{2}\left(3 \nabla_{i} G_{j}+G^{\alpha} \nabla_{i} \nabla_{j} \nabla_{k} G_{\alpha}\right) \\
= & \left(m_{3 / 2}-F^{\alpha} \partial_{\alpha} \log m_{3 / 2}\right) Y_{i j k}+F^{\alpha} \partial_{\alpha} Y_{i j k}-3 F^{\alpha} \Gamma_{\alpha(i}^{l} Y_{l j k)}, \\
B_{i j}= & m_{3 / 2}^{2}\left(2 \nabla_{i} G_{j}+G^{\alpha} \nabla_{i} \nabla_{j} G_{\alpha}\right)=2 m_{3 / 2}^{2} Z_{i j}-m_{3 / 2} F^{\bar{\alpha}} \partial_{\bar{\alpha}} Z_{i j} \\
& +m_{3 / 2} F^{\alpha}\left(\partial_{\alpha} Z_{i j}-\Gamma_{\alpha i}^{k} Z_{k j}-\Gamma_{\alpha j}^{k} Z_{k i}\right)-F^{\alpha} F^{\bar{\beta}}\left(Z_{i j \alpha \bar{\beta}}-\Gamma_{\alpha i}^{k} Z_{k j \bar{\beta}}-\Gamma_{\alpha j}^{k} Z_{k i \bar{\beta}}\right) \\
& -e^{\frac{\mathcal{K}}{2}} \tilde{\mu}_{i j} m_{3 / 2}+e^{\frac{\mathcal{K}}{2}} F^{\alpha}\left(\partial_{\alpha} \tilde{\mu}_{i j}+\frac{1}{2} \hat{K}_{\alpha} \tilde{\mu}_{i j}-\Gamma_{\alpha i}^{l} \tilde{\mu}_{l j}-\Gamma_{\alpha j}^{l} \tilde{\mu}_{i l}\right), \\
M_{1 / 2}^{a}= & \frac{1}{2} g_{a}^{2} F^{\alpha} \partial_{\alpha} f_{a},
\end{aligned}
$$

where $\alpha, \beta$ are hidden sector supersymmetry breaking indices, $f_{a}$ is the gauge kinetic function for the gauge group factor $G_{a}$ and some basic definitions are given in the appendix. For geometrical separation in the internal space (sequestering) or in no-scale like models, tree level soft masses are zero or highly suppressed and one-loop contributions become relevant. One loop contributions to gaugino masses, called anomaly-mediated contributions in $[67,68]$, are given in general by $[69]$

$$
M_{1 / 2}^{a}=-\frac{g_{a}^{2}}{16 \pi^{2}}\left\{\left(3 T_{G}^{a}-T_{R}^{a}\right) m_{3 / 2}+\left(T_{G}^{a}-T_{R}^{a}\right) K_{\alpha} F^{\alpha}+\frac{2 T_{R}^{a}}{d_{R}}\left(\log \operatorname{det} K_{i \bar{\jmath}, R^{a}}\right)_{\alpha} F^{\alpha}\right\},
$$

where $T_{G}^{a}$ is the Dynkin index for the adjoint representation of the gauge group and $T_{G}^{a}$, $K_{i \bar{j}, R^{a}}$ are the Dynkin index for the chiral matter fields in representation $R^{a}$ and their Kähler metric, respectively. The complete one-loop expression for the other soft terms is more involved [70]. In the limit of small hidden-sector vev's, the one-loop induced anomaly mediated contributions take forms of the type $m_{\text {soft }} \sim\left(b g^{2}\right) /\left(16 \pi^{2}\right) m_{3 / 2}$, depending on beta functions $b$ (and anomalous dimensions) of the low-energy spectrum.

On general grounds, for matter fields which are not sequestered from the KS throat, one expects soft masses of order $m_{3 / 2}$. For fields far away from the throat, from the fourdimensional perspective, one could anticipate soft terms generated by the Kähler modulus contribution to supersymmetry breaking $F^{\rho}$ and one-loop anomaly-mediated contributions. These two contributions are similar in size, as emphasized in [71, 72] and parametrically (one-loop) suppressed compared to the gravitino mass. 


\subsection{Distant D3 "matter" branes}

One test of the action (3.3) is to add distant, from the throat, D3 "matter" branes. Denoting by $Q_{i}$ the (distant) D3 brane superfields, the tree-level action in type IIB orientifolds including them was derived in $[75,76]$. In the absence of warping, the Kähler and the complex structure moduli spaces do not talk to each other, except for the holomorphic terms of coefficients called $z_{i j}$ below. The new ingredient from the warping is that the main contribution to the complex structure field $Z$ Kähler potential changes drastically (in the absence of matter fields) according to the previous sections, whereas the perturbative contribution is negligible. Taking this into account, we arrive at the following effective action

$$
\begin{aligned}
\mathcal{K} & =-3 \log \left(\rho+\bar{\rho}-\frac{|X|^{2}}{3}-\frac{\xi|Z|^{2 / 3}}{3}-\left|Q_{i}\right|^{2}-z_{i j}^{\prime}\left(\bar{Z} Q_{i} Q_{j}+\text { h.c. }\right)\right) \\
& =-3 \log \left(\rho+\bar{\rho}-\frac{|X|^{2}}{3}-|Y|^{2}-\left|Q_{i}\right|^{2}-z_{i j}\left(\bar{Y}^{3} Q_{i} Q_{j}+\text { h.c. }\right)\right) \equiv-3 \log r, \\
W & =W_{1}(\rho, Y, X)+W_{2}\left(Q_{i}\right) .
\end{aligned}
$$

Notice that (4.5) has a sequestered form, except the terms in $z_{i j}$. Using the appendix, it can be shown that in this case the Riemann tensor satisfies the identity

$$
R_{i \bar{\jmath} \alpha \bar{\beta}}=\frac{1}{3} G_{i \bar{\jmath}} G_{\alpha \bar{\beta}},
$$

where $\alpha, \beta=X, Z, \rho$ are moduli indices and $i, j$ are matter indices, such that the tree-level scalar soft masses $m_{i j}^{2}$ in (4.3) vanish after reinforcing the cancelation of the cosmological constant $G^{\alpha} G_{\alpha}=3$. The tree-level A-terms can be written more explicitly as

$$
A_{i j k}=m_{3 / 2}^{2}\left[3 G_{i j k}+G^{\alpha}\left(\partial_{\alpha} G_{i j k}-\Gamma_{i \alpha}^{m} G_{j k m}-\Gamma_{j \alpha}^{m} G_{i k m}-\Gamma_{k \alpha}^{m} G_{i j m}\right)\right]
$$

and they turn out to also vanish for zero cosmological constant.

The tree-level supersymmetric masses in our case are given by

$$
\mu_{i j}=m_{3 / 2}\left(\partial_{i} G_{j}-\Gamma_{i j}^{\alpha} G_{\alpha}\right)=-2 z_{i j} \bar{y}^{2}\left(2 \bar{y} W_{T}+3 W_{y}\right) e^{\frac{\mathcal{K}}{2}} \sim \mathcal{O}\left(\frac{Y_{0}^{4}}{\rho_{0}^{3 / 2}}\right) \sim \mathcal{O}\left(\frac{Z_{0}^{4 / 3}}{\rho_{0}^{3 / 2}}\right) .
$$

This result is in qualitative agreement with the action obtained from the compactification worked out in $[75,76]$. It is however important to remember that our effective action is only valid in the leading order in an expansion in powers of $Z^{2 / 3}$. The leading order contribution to the $\mu$-terms is small and subleading and probably a mass of order $Z^{4 / 3}$ is quantitatively not fully under control. The $B \mu$-like terms $B_{i j}$ in (4.3) are of order $\mathcal{O}\left(Y^{6}\right) \sim \mathcal{O}\left(Z^{2}\right)$, but probably their computation is also not fully reliable. It is also possible that the coefficients $z_{i j}$ are warped down such that the real values of $\mu_{i j}, B_{i j}$ is even smaller than the one we estimated here.

The tree-level action of the distant D3 branes seem to realize therefore, at the leading order in a power expansion of $S^{2 / 3}$, an approximate sequestered case [67, 68] where all tree-level "soft terms" for matter D3 fields are zero (or very small). However, it was 
argued in $[73,74]$ that sequestering is broken by the coupling of D3 branes to the gaugino condensates on D7 branes or on Euclidian D3 branes providing the nonperturbative superpotential for the Kähler modulus $\rho$. This effect, coming from threshold corrections, breaks the sequestered form of the superpotential (4.5), by generating terms of the type $w(Q) e^{-a \rho}$. Such terms generate soft terms, but not non-holomorphic soft scalar masses. On the other hand, it was shown in [71-74] that small values of the latter, suppressed with respect to the gravitino mass, are also generated by the antibrane uplift. In our manifestly supersymmetric nilpotent formalism, such terms can be generated by breaking sequestering in the Kähler potential. Breaking sequestering and agreement with previous results require therefore a modification of our effective action (4.5) by

$$
\Delta K=\frac{D|X|^{2} \bar{Q}_{i} Q_{i}}{r^{4}}, \quad \Delta W=w\left(Q_{i}\right) e^{-a \rho},
$$

where $D$ is a constant and $w\left(Q_{i}\right)$ a holomorphic gauge invariant function of matter chiral fields. The form of $\Delta K$, which should be treated as a small perturbation of the Kähler potential in (4.5), is determined by matching the resulting generated non-holomorphic scalar masses to previous results, $m_{0}^{2} \sim m_{3 / 2}^{2} /(a r)^{2}$ for the canonically normalized matter field [71-74]. The resulting soft terms are suppressed and similar in size to the anomaly mediated terms discussed below. It would be interesting to understand from first principles the term $\Delta K$ breaking sequestering.

Notice that the $\overline{\mathrm{D} 3}$ brane interacts via loops of open strings, or equivalently, by treelevel exchange of closed strings, with distant D3 branes. This is a higher-dimensional tiny branes-antibrane interaction which is actually a one-loop effect, that has to be added to the effective action above. The sequestering is approximative therefore from several viewpoints: the threshold effects on D7 branes (or Euclidian D3 branes) and the antibrane-D3 brane distant interactions. In addition, there are small distant branes mass terms related to the contribution to supersymmetry breaking of the conifold field $Z$. They could maybe have an interpretation since $F^{Z} \neq 0$ has an effect similar to the generation of an effective $(1,2)$ flux.

From a four-dimensional perspective, one can also contemplate calculating the one-loop anomaly-mediated type contributions. For the effective action (4.5), the one-loop gaugino masses reduce at leading order to the universal term

$$
M_{1 / 2}^{a}=-\frac{g_{a}^{2}}{16 \pi^{2}}\left(3 T_{G}^{a}-T_{R}^{a}\right)\left(m_{3 / 2}+\frac{1}{3} K_{\alpha} F^{\alpha}\right) \simeq-\frac{g_{a}^{2}}{16 \pi^{2}}\left(3 T_{G}^{a}-T_{R}^{a}\right) m_{3 / 2}
$$

\subsection{D3 branes-antibrane interactions: a higher-dimensional perspective}

In addition to the threshold effects on D7 branes or euclidian D3 branes, the sequestering is broken explicitly by the distant branes-antibrane interactions, which generate an interaction between the antibrane and the distant antibranes [46]. This interaction can be described by a correction to the Kähler potential, breaking the sequestered structure, of the type

$$
\Delta \mathcal{K}=\frac{\xi_{z}^{\prime}|Z|^{4 / 3}|X|^{2}}{r\left(\mathbf{r}_{\mathbf{0}}+\mathbf{Q}\right)^{4}}
$$


where $d^{2}=\mathbf{r}_{\mathbf{0}}{ }^{2}$ is the radial distance between the D3 branes and the antibrane. Indeed, for $\mathbf{Q}_{i}=0$ this term changes the metric $\mathcal{K}_{X \bar{X}}=\left(1+\frac{\xi_{z}^{\prime}|Z|^{4 / 3}}{d^{4}}\right) \frac{1}{r}$ and changes the scalar potential generated by the uplift sector to

$$
\begin{aligned}
V_{\overline{\mathrm{D} 3}}+V_{\mathrm{D} 3-\overline{\mathrm{D}} 3}=\mathcal{K}^{X \bar{X}} e^{\mathcal{K}}\left|D_{X} W\right|^{2} & \simeq \frac{c^{\prime \prime}|Z|^{4 / 3}}{\pi(\rho+\bar{\rho})^{2} g_{s} M^{2}}\left(1-\frac{\xi_{z}^{\prime}|Z|^{4 / 3}}{d^{4}}\right) \\
& \equiv 2 T_{3} H^{-1}\left(r_{0}\right)\left[1-\frac{1}{N} \frac{H\left(r_{1}\right)}{H\left(r_{0}\right)}\right]
\end{aligned}
$$

where $r_{0}\left(r_{1}\right)$ is the radial position of the $\overline{\mathrm{D} 3}$ brane (matter $\left.D 3\right)$ branes and we performed a leading-order expansion in powers of $Z \ll 1$. An expansion in powers of the matter fields gives the antibrane-distant D3 branes fields interaction

$$
V_{\mathrm{D} 3-\overline{\mathrm{D}} 3}=-\frac{\xi_{z}^{\prime} c^{\prime \prime}|Z|^{8 / 3}}{\pi r^{2} d^{4} g_{s} M^{2}}\left[1-\frac{4 \mathbf{r}_{\mathbf{0}} \mathbf{Q}}{d^{2}}-\frac{2 \mathbf{Q}^{2}}{d^{2}}+\frac{12\left(\mathbf{r}_{\mathbf{0}} \mathbf{Q}\right)^{2}}{d^{4}}+\cdots\right] .
$$

In the higher-dimensional approach, the second derivative of this scalar potential gives the mass matrix for $\mathbf{Q}_{i}$, which lead to tachyonic directions [77]. It is however useful to cast the problem as a correction to the $4 \mathrm{~d}$ Kähler potential. By using the dimension-1 field $Y$, it is given by

$$
\Delta \mathcal{K}=\frac{\xi^{\prime}|Y|^{4}|X|^{2}}{r d^{4}}\left[1-\frac{4 \mathbf{r}_{\mathbf{0}} \mathbf{Q}}{d^{2}}-\frac{2 \mathbf{Q}^{2}}{d^{2}}+\frac{12\left(\mathbf{r}_{\mathbf{0}} \mathbf{Q}\right)^{2}}{d^{4}}+\cdots\right],
$$

where $\xi^{\prime}=\left(3 c^{\prime} g_{s} M^{2}\right)^{-2} \xi_{z}^{\prime}$. The linear term signifies that the distant D3 brane does not sit at an extremum, which can be remedied, in case of orientifolds, by adding an image brane at $-\mathbf{r}_{\mathbf{0}}$. The quadratic terms are more conveniently written in a complex basis $\Phi_{i}, z_{i}$, $i=1,2,3$, by introducing $\Phi_{1}=\frac{1}{\sqrt{2}}\left(Q_{1}+i Q_{2}\right), z_{1}=\frac{1}{\sqrt{2}}\left(y_{1}+i y_{2}\right)$, etc. One gets

$$
\Delta \mathcal{K}=\frac{\xi^{\prime}|Y|^{4}|X|^{2}}{4 r|z|^{4}}\left[1-\frac{2}{|z|^{2}}\left(\delta_{i \bar{\jmath}}-\frac{3 z_{i} \bar{z}_{\bar{\jmath}}}{4|z|^{2}}\right) \bar{\Phi}^{i} \Phi^{\bar{\jmath}}+\frac{3}{4|z|^{4}}\left(\bar{z}_{\bar{\imath}} \bar{z}_{\bar{\jmath}} \Phi^{\bar{\imath}} \Phi^{\bar{\jmath}}+\text { h.c. }\right)+\cdots\right] .
$$

The non-holomorphic piece $\bar{\Phi}_{i} \Phi_{j}$ changes the metric for matter fields and changes the Riemann tensor of the Kähler manifold $R=R^{(0)}+R^{(1)}$. It generates an additional contribution to the soft scalar masses

$$
m_{i \bar{\jmath}}^{2}=-m_{3 / 2}^{2}\left|G^{X}\right|^{2} R_{i \bar{\jmath} X \bar{X}}^{(1)}=-\left|F^{X}\right|^{2} R_{i \bar{\jmath} X \bar{X}}^{(1)}=\left|F^{X}\right|^{2} \frac{\xi^{\prime}|Y|^{4}}{2 r|z|^{6}}\left(\delta_{i \bar{\jmath}}-\frac{3 z_{i} \bar{z}_{\bar{\jmath}}}{4|z|^{2}}\right)
$$

which are positive definite and of order $m_{0}^{2} \sim Y_{0}^{8} /\left(\rho_{0}^{2}|z|^{6}\right)$, in agreement with the masses computed from the higher-dimensional potential (4.13). The holomorphic soft masses are then given by

$$
B_{i j}=\left|F^{X}\right|^{2} \frac{3 \xi^{\prime}|Y|^{4}}{16 r|z|^{8}} z_{i} z_{j} .
$$

Notice that they are smaller $\left(\mathcal{O}\left(Y^{8}\right)\right)$ than the tree-level $4 \mathrm{~d}$ supergravity computation of the previous section. They are of the same order than the non-holomorphic masses $m_{i \bar{\jmath}}^{2}$ 
and generate tachyonic masses after diagonalization [77]. However, in a realistic compactification the distant D3 branes have to experience additional projections of the spectrum in order to make it compatible with a MSSM one. For example, it should sit at an orbifold or orientifold singularity. If this is achieved, the only sector that can have $B$ terms is the Higgs sector. A Higgs vev could lead to correct electroweak symmetry breaking if its value is in the $\mathrm{TeV}$ range, therefore if the soft terms are in the TeV range. The size of soft terms provided by (4.16)-(4.17) can be re-expressed as a function of the gravitino mass and the distance from the D3 antibrane. Their typical size is then worked out to be of order $m_{\text {soft }} \sim\left(\frac{\rho_{0}}{|z|}\right)^{3} \frac{m_{3 / 2}^{2}}{M_{P}}$. If the one-loop contributions would be smaller, intermediate values of $m_{3 / 2}$ can lead therefore to $\mathrm{TeV}$ values for masses. However, as discussed in the previous section, from a four-dimensional perspective, threshold corrections on D7 branes or Euclidian D3 branes and other one-loop corrections are expected to break sequestering and generate suppressed soft terms [71-74] with respect to the gravitino mass, similar in size to anomaly mediated contributions [67,68]. Their size is typically a one-loop factor times $m_{3 / 2}$ and will therefore dominate over the (smaller) contributions discussed above. It would be interesting to evaluate from a higher-dimensional perspective, based on antibrane-distant branes interactions, the one-loop generated soft terms and compare them to the four-dimensional expressions. This is however beyond the scope of the current letter.

Notice that, if one uses some distant D3 branes for inflation, then a scalar potential of the type (4.12) becomes the inflationary energy scale. From the current bounds on the tensor to scalar ratio $r \leq 0.1$, one gets another bound on the acceptable values of $Z_{0}$

$$
V_{\mathrm{inf}}=V_{\mathrm{D} 3-\overline{\mathrm{D} 3}} \leq 10^{16} \mathrm{GeV} \quad \rightarrow \quad \frac{Z_{0}^{4 / 3}}{\rho_{0}^{2}} \leq 10^{-9} .
$$

For a KKLT type scenario, this implies $Z_{0} \leq 10^{-5}$ and therefore again a largish contribution to the localized D3 flux $\left|Q_{3}^{\text {loc }}\right|>\mathcal{O}(80)$, for $\Lambda_{0} \sim M_{P}$.

\subsection{4d versus higher-dimensional description}

It was shown in [31] that Kaluza-Klein states localized on the KS throat have masses parametrically of the same order as the mass of the field $Z$. Then a full $4 \mathrm{~d}$ description of the dynamics is probably not an accurate approximation, unless one goes to low energies and integrates the whole KK tower and the field $Z$. This it difficult to do in practice. On the other hand, the gravitino mass is small enough that a supergravity description should exist at low-energy. It is difficult to make definite statements, but some qualitative remarks go as follows.

From a $4 \mathrm{~d}$ perspective, assuming that the $4 \mathrm{~d}$ SUGRA description is valid, one can use the general formulae of soft terms as in the beginning of the previous section. Alternatively, we can use higher-dimensional brane-antibrane forces as a starting point of computing soft terms. At tree-level, we found qualitative agreement for distant (from the throat) D3 branes, which seem to fulfill an approximate sequestering, broken by threshold effects that we commented around (4.9). Additional contributions come from small mass terms related to the contribution to supersymmetry breaking of the conifold field $Z$, which has 
an effect similar to an effective $(1,2)$ flux. As mentioned previously, it is also plausible that the warping is suppressing further the real values of these masses. At one-loop level, $4 \mathrm{~d}$ anomaly-mediated contributions to soft terms are present, consistent with a diffuse transmission of supersymmetry breaking across the bulk. Their higher-dimensional origin is not clear and it would be very interesting to check them in detail from the higherdimensional viewpoint, in particular the one-loop contribution to the gaugino masses. We hope to be able to return to this issue elsewhere.

\section{Conclusions}

We continued the analysis of the KKLT moduli stabilization with antibrane uplift in the context of the Klebanov-Strassler warped compactification, taking into account the light complex structure field $S(Z)$ identified in [30,31]. Assuming the validity of the 4 d supergravity description and using a manifestly supersymmetric formulation of the uplift via a nilpotent field on the antibrane, we worked out the vacuum structure and physical spectrum, confirming the potential destabilization of the KS throat. The minimal value of the needed flux (1.1), combined with needed redshift for various physical purposes, translates into (relatively) large values of the localized D3 charge $Q_{3}^{\text {loc }}$, beyond the usual perturbative values $Q_{3}^{\text {loc }} \leq 16$. Notice that this is not a surprise in itself. Indeed, the gravitational KS solution is valid for $g_{s} M \gg 1$, which is generically stronger than our destabilization limit $\sqrt{g_{s}} M \geq 6.8$. However, using just the standard KS validity bound leads to weaker limits on $Q_{3}^{\text {loc }}$ by choosing maximal values $\left(g_{s} \sim \mathcal{O}(1)\right)$ of the string coupling, which are reasonable at least in F-theory. The destabilization bound, on the other hand, leads to limits on $Q_{3}^{\text {loc }}$ which are independent on the string coupling and feature generically large numbers. Our viewpoint in this paper is that, whereas it could be difficult to obtain such large localized D3 charges in perturbative type II strings, it is presumably possible in F-theory. In such F-theory models, however, the stabilization of a large number of complex structure moduli is challenging [58].

We studied the consequences of including the light complex structure modulus $Z$ in the low-energy description for the vacuum structure and phenomenology. The effect of the antibrane uplift is that the vev of $Z$ is shifted such that its contribution to supersymmetry breaking is large, comparable with that of the nilpotent field. Secondly, whereas the conifold field is generically heavier than the volume modulus, justifying an integrating out procedure, this is not always the case and there is a condition that the flux and the other parameters have to satisfy. Most of supersymmetry breaking is localized at the tip of the throat, but a small amount of supersymmetry breaking is transmitted across the bulk far from the throat. We studied the effects of the supersymmetry breaking on observable sector fields, in particular on distant D3 branes. Neglecting threshold effects, we found an approximate sequestering, with the only non-zero masses in the observable sector are of $\mu, B \mu$ type and very small. Their appearance could be due to the fact that the complex structure modulus $Z$ contributes to supersymmetry breaking, which acts effectively as an $(1,2)$ flux. Comparing the results from a four-dimensional supergravity perspective and from a higher-dimensional one, one finds qualitative agreement at tree-level. Threshold ef- 
fects (4.9) are expected to add other contributions breaking sequestering [71-74]. It would be interesting to understand the origin of the term $\Delta K$ in (4.9) in the nilpotent formalism, needed in order to match non-holomorphic soft scalar masses with previous results.

It would be clearly interesting to investigate further one-loop soft terms from four dimensional and higher-dimensional perspective and check their compatibility. It would also interesting to study from a similar viewpoint other models of moduli stabilization and check if the potential throat destabilization still exists. The existence of a critical value of the flux to avoid destabilization could be addressed directly in the dual gauge theory of the KS throat. Another direction to investigate is the search of alternatives, to the $\overline{\mathrm{D} 3}$ antibrane, uplifts of the vacuum energy. Finally, we commented on the dS swampland conjecture [59-61]. It would be interesting to investigate further this refined model of moduli stabilization from the viewpoint of the other swampland conjectures [78-81] (for an extensive review and references, see [82]).

Note added. While this paper was completed, the paper [83] appeared, which has some overlap with ours and interpret our normalized field $Y$ of section 3 with the radion from a $5 \mathrm{~d}$ perspective. We thank Lisa Randall for discussions and for sharing her preliminary draft.

\section{Acknowledgments}

We are grateful to Iosif Bena and Mariana Graña for collaboration in an earlier stage of this work and many important discussions. We would like to thank Ralph Blumenhagen, Arthur Hebecker, Luca Martucci and Lisa Randall for very helpful discussions and correspondence. This work was supported in part by the ANR grant Black-dS-String ANR-16-CE31-0004-01.

\section{A Supergravity formulae}

As well-known, the $4 \mathrm{~d}$ supergravity action does not depend separately on $W$ and $\mathcal{K}$, but on the function $G=\mathcal{K}+\log |W|^{2}$. Some useful formulae for a Kähler space, for which $G_{I \bar{J}}=K_{I \bar{J}}=\partial_{I} \partial_{\bar{J}} \mathcal{K}$, used in the text and in particular to evaluate (4.3) are

$$
\begin{aligned}
G^{I} & =G^{I \bar{J}} G_{\bar{J}}, \quad \Gamma_{I J}^{K}=G^{K \bar{M}} \partial_{I} G_{J \bar{M}}, \quad \nabla_{I} V_{J}=\partial_{I} V_{J}-\Gamma_{I J}^{K} V_{K}, \\
R_{I \bar{J} K \bar{L}} & =\partial_{K} \partial_{\bar{L}} G_{I \bar{J}}-G^{M \bar{N}} \partial_{K} G_{I \bar{N}} \partial_{\bar{L}} G_{M \bar{J}} .
\end{aligned}
$$

In the case of our effective action (4.5), the nontrivial components of the connections are $(i, j$ denote matter fields indices in what follows)

$$
\Gamma_{i \rho}^{j}=-\frac{1}{r} \delta_{i}^{j}, \quad \Gamma_{i y}^{j}=\frac{\bar{y}}{r} \delta_{i}^{j}, \quad \Gamma_{i j}^{\rho}=4 z_{i j} \bar{y}^{3}, \quad \Gamma_{i j}^{y}=6 z_{i j} \bar{y}^{2} .
$$

By using the metric components and (A.1), (A.2), one can easily verify

$$
R_{i \bar{\jmath} \alpha \bar{\beta}}=\frac{1}{3} G_{i \bar{\jmath}} G_{\alpha \bar{\beta}},
$$


where $\alpha, \beta=X, Y, \rho$ and $i, j$ are matter fields indices. In the presence of the correction to the Kähler potential (4.9) breaking sequestering, there is a new correction to the Riemann tensor, which becomes, at first order in the correction,

$$
R_{i \bar{\jmath} \alpha \bar{\beta}}=\frac{1}{3}\left(1+\frac{D}{r^{2}} \delta_{\alpha X} \delta_{\bar{\beta} \bar{X}}\right) G_{i \bar{\jmath}} G_{\alpha \bar{\beta}} .
$$

Analogously, for the correction in (4.11), the Riemann tensor becomes

$$
R_{i \bar{\jmath} \alpha \bar{\beta}}=\frac{1}{3}\left(G_{\alpha \bar{\beta}}-\frac{2 \xi^{\prime}\left|Y_{0}\right|^{4}}{N d^{6}} \delta_{\alpha X} \delta_{\bar{\beta} \bar{X}}\right) G_{i \bar{\jmath}} .
$$

Open Access. This article is distributed under the terms of the Creative Commons Attribution License (CC-BY 4.0), which permits any use, distribution and reproduction in any medium, provided the original author(s) and source are credited.

\section{References}

[1] S. Kachru, R. Kallosh, A.D. Linde and S.P. Trivedi, de Sitter vacua in string theory, Phys. Rev. D 68 (2003) 046005 [hep-th/0301240] [INSPIRE].

[2] S. Sugimoto, Anomaly cancellations in type-I D9-D $\overline{9}$ system and the USp(32) string theory, Prog. Theor. Phys. 102 (1999) 685 [hep-th/9905159] [InSPIRE].

[3] I. Antoniadis, E. Dudas and A. Sagnotti, Brane supersymmetry breaking, Phys. Lett. B 464 (1999) 38 [hep-th/9908023] [INSPIRE].

[4] C. Angelantonj, Comments on open string orbifolds with a nonvanishing $B_{a b}$, Nucl. Phys. B 566 (2000) 126 [hep-th/9908064] [INSPIRE].

[5] G. Aldazabal and A.M. Uranga, Tachyon free nonsupersymmetric type IIB orientifolds via Brane-antibrane systems, JHEP 10 (1999) 024 [hep-th/9908072] [INSPIRE].

[6] C. Angelantonj, I. Antoniadis, G. D'Appollonio, E. Dudas and A. Sagnotti, Type I vacua with brane supersymmetry breaking, Nucl. Phys. B 572 (2000) 36 [hep-th/9911081] [INSPIRE].

[7] E. Dudas and J. Mourad, Consistent gravitino couplings in nonsupersymmetric strings, Phys. Lett. B 514 (2001) 173 [hep-th/0012071] [INSPIRE].

[8] G. Pradisi and F. Riccioni, Geometric couplings and brane supersymmetry breaking, Nucl. Phys. B 615 (2001) 33 [hep-th/0107090] [INSPIRE].

[9] I. Bena, M. Graña and N. Halmagyi, On the Existence of Meta-stable Vacua in Klebanov-Strassler, JHEP 09 (2010) 087 [arXiv: 0912.3519] [INSPIRE].

[10] I. Bena, G. Giecold, M. Graña, N. Halmagyi and S. Massai, On Metastable Vacua and the Warped Deformed Conifold: Analytic Results, Class. Quant. Grav. 30 (2013) 015003 [arXiv: 1102.2403] [INSPIRE].

[11] I. Bena, G. Giecold, M. Graña, N. Halmagyi and S. Massai, The backreaction of anti-D3 branes on the Klebanov-Strassler geometry, JHEP 06 (2013) 060 [arXiv:1106.6165] [INSPIRE].

[12] I. Bena, M. Graña, S. Kuperstein and S. Massai, Anti-D3 Branes: Singular to the bitter end, Phys. Rev. D 87 (2013) 106010 [arXiv:1206.6369] [INSPIRE]. 
[13] I. Bena, M. Graña, S. Kuperstein and S. Massai, Tachyonic Anti-M2 Branes, JHEP 06 (2014) 173 [arXiv: 1402.2294] [INSPIRE].

[14] I. Bena, M. Graña, S. Kuperstein and S. Massai, Giant Tachyons in the Landscape, JHEP 02 (2015) 146 [arXiv: 1410.7776] [inSPIRE].

[15] B. Michel, E. Mintun, J. Polchinski, A. Puhm and P. Saad, Remarks on brane and antibrane dynamics, JHEP 09 (2015) 021 [arXiv: 1412.5702] [INSPIRE].

[16] I. Bena, J. Blåbäck and D. Turton, Loop corrections to the antibrane potential, JHEP 07 (2016) 132 [arXiv: 1602.05959] [INSPIRE].

[17] D. Cohen-Maldonado, J. Diaz, T. van Riet and B. Vercnocke, Observations on fluxes near anti-branes, JHEP 01 (2016) 126 [arXiv:1507.01022] [INSPIRE].

[18] D. Cohen-Maldonado, J. Diaz and F.F. Gautason, Polarised antibranes from Smarr relations, JHEP 05 (2016) 175 [arXiv: 1603.05678] [INSPIRE].

[19] J. Armas, N. Nguyen, V. Niarchos, N.A. Obers and T. Van Riet, Meta-stable non-extremal anti-branes, Phys. Rev. Lett. 122 (2019) 181601 [arXiv:1812.01067] [INSPIRE].

[20] J. Blåbäck, F.F. Gautason, A. Ruipérez and T. Van Riet, Anti-brane singularities as red herrings, JHEP 12 (2019) 125 [arXiv:1907.05295] [INSPIRE].

[21] S. Sethi, Supersymmetry Breaking by Fluxes, JHEP 10 (2018) 022 [arXiv:1709.03554] [INSPIRE].

[22] J. Moritz, A. Retolaza and A. Westphal, Toward de Sitter space from ten dimensions, Phys. Rev. D 97 (2018) 046010 [arXiv:1707.08678] [InSPIRE].

[23] Y. Hamada, A. Hebecker, G. Shiu and P. Soler, On brane gaugino condensates in 10d, JHEP 04 (2019) 008 [arXiv: 1812.06097] [INSPIRE].

[24] R. Kallosh, Gaugino Condensation and Geometry of the Perfect Square, Phys. Rev. D 99 (2019) 066003 [arXiv:1901.02023] [INSPIRE].

[25] Y. Hamada, A. Hebecker, G. Shiu and P. Soler, Understanding KKLT from a $10 d$ perspective, JHEP 06 (2019) 019 [arXiv:1902.01410] [INSPIRE].

[26] F. Carta, J. Moritz and A. Westphal, Gaugino condensation and small uplifts in KKLT, JHEP 08 (2019) 141 [arXiv:1902.01412] [INSPIRE].

[27] F.F. Gautason, V. Van Hemelryck, T. Van Riet and G. Venken, A 10d view on the KKLT AdS vacuum and uplifting, JHEP 06 (2020) 074 [arXiv: 1902.01415] [INSPIRE].

[28] I. Bena, M. Graña, N. Kovensky and A. Retolaza, Kähler moduli stabilization from ten dimensions, JHEP 10 (2019) 200 [arXiv:1908.01785] [INSPIRE].

[29] S. Kachru, M. Kim, L. McAllister and M. Zimet, de Sitter Vacua from Ten Dimensions, arXiv: 1908.04788 [INSPIRE].

[30] I. Bena, E. Dudas, M. Graña and S. Lüst, Uplifting Runaways, Fortsch. Phys. 67 (2019) 1800100 [arXiv: 1809.06861] [INSPIRE].

[31] R. Blumenhagen, D. Kläwer and L. Schlechter, Swampland Variations on a Theme by KKLT, JHEP 05 (2019) 152 [arXiv: 1902.07724] [INSPIRE].

[32] I. Bena, A. Buchel and S. Lüst, Throat destabilization (for profit and for fun), arXiv:1910.08094 [INSPIRE].

[33] M. Roček, Linearizing the Volkov-Akulov Model, Phys. Rev. Lett. 41 (1978) 451 [InSPIRE]. 
[34] U. Lindström and M. Roček, Constrained Local Superfields, Phys. Rev. D 19 (1979) 2300 [INSPIRE].

[35] R. Casalbuoni, S. De Curtis, D. Dominici, F. Feruglio and R. Gatto, Nonlinear Realization of Supersymmetry Algebra From Supersymmetric Constraint, Phys. Lett. B 220 (1989) 569 [INSPIRE].

[36] Z. Komargodski and N. Seiberg, From Linear SUSY to Constrained Superfields, JHEP 09 (2009) 066 [arXiv:0907.2441] [INSPIRE].

[37] I. Antoniadis, E. Dudas, S. Ferrara and A. Sagnotti, The Volkov-Akulov-Starobinsky supergravity, Phys. Lett. B $\mathbf{7 3 3}$ (2014) 32 [arXiv: 1403.3269] [InSPIRE].

[38] S. Ferrara, R. Kallosh and A. Linde, Cosmology with Nilpotent Superfields, JHEP 10 (2014) 143 [arXiv: 1408.4096] [INSPIRE].

[39] M. Graña and J. Polchinski, Supersymmetric three form flux perturbations on AdS $S_{5}$, Phys. Rev. D 63 (2001) 026001 [hep-th/0009211] [INSPIRE].

[40] S.B. Giddings, S. Kachru and J. Polchinski, Hierarchies from fluxes in string compactifications, Phys. Rev. D 66 (2002) 106006 [hep-th/0105097] [INSPIRE].

[41] L. Randall and R. Sundrum, A large mass hierarchy from a small extra dimension, Phys. Rev. Lett. 83 (1999) 3370 [hep-ph/9905221] [INSPIRE].

[42] L. Randall and R. Sundrum, An alternative to compactification, Phys. Rev. Lett. 83 (1999) 4690 [hep-th/9906064] [INSPIRE].

[43] I.R. Klebanov and M.J. Strassler, Supergravity and a confining gauge theory: Duality cascades and $\chi b$-resolution of naked singularities, JHEP 08 (2000) 052 [hep-th/0007191] [INSPIRE].

[44] M.R. Douglas, J. Shelton and G. Torroba, Warping and supersymmetry breaking, arXiv:0704. 4001 [INSPIRE].

[45] M.R. Douglas and G. Torroba, Kinetic terms in warped compactifications, JHEP 05 (2009) 013 [arXiv: 0805.3700] [INSPIRE].

[46] S. Kachru, R. Kallosh, A.D. Linde, J.M. Maldacena, L.P. McAllister and S.P. Trivedi, Towards inflation in string theory, JCAP 10 (2003) 013 [hep-th/0308055] [INSPIRE].

[47] A. Buchel, Klebanov-Strassler black hole, JHEP 01 (2019) 207 [arXiv:1809. 08484] [INSPIRE].

[48] E.A. Bergshoeff, K. Dasgupta, R. Kallosh, A. Van Proeyen and T. Wrase, $\overline{\mathrm{D} 3}$ and $d S, J H E P$ 05 (2015) 058 [arXiv: 1502.07627] [INSPIRE].

[49] R. Kallosh, F. Quevedo and A.M. Uranga, String Theory Realizations of the Nilpotent Goldstino, JHEP 12 (2015) 039 [arXiv:1507.07556] [INSPIRE].

[50] I. García-Etxebarria, F. Quevedo and R. Valandro, Global String Embeddings for the Nilpotent Goldstino, JHEP 02 (2016) 148 [arXiv: 1512.06926] [INSPIRE].

[51] M.P. Garcia del Moral, S. Parameswaran, N. Quiroz and I. Zavala, Anti-D3 branes and moduli in non-linear supergravity, JHEP 10 (2017) 185 [arXiv:1707.07059] [INSPIRE].

[52] N. Cribiori, C. Roupec, T. Wrase and Y. Yamada, Supersymmetric anti-D3-brane action in the Kachru-Kallosh-Linde-Trivedi setup, Phys. Rev. D 100 (2019) 066001 [arXiv: 1906.07727] [INSPIRE]. 
[53] A.M. Uranga, Comments on nonsupersymmetric orientifolds at strong coupling, JHEP 02 (2000) 041 [hep-th/9912145] [INSPIRE].

[54] O. DeWolfe and S.B. Giddings, Scales and hierarchies in warped compactifications and brane worlds, Phys. Rev. D 67 (2003) 066008 [hep-th/0208123] [INSPIRE].

[55] S.B. Giddings and A. Maharana, Dynamics of warped compactifications and the shape of the warped landscape, Phys. Rev. D 73 (2006) 126003 [hep-th/0507158] [INSPIRE].

[56] C.P. Burgess et al., Warped Supersymmetry Breaking, JHEP 04 (2008) 053 [hep-th/0610255] [INSPIRE].

[57] G. Shiu, G. Torroba, B. Underwood and M.R. Douglas, Dynamics of Warped Flux Compactifications, JHEP 06 (2008) 024 [arXiv:0803.3068] [INSPIRE].

[58] I. Bena, J. Blåbäck, M. Graña and S. Lüst, The Tadpole Problem, arXiv:2010.10519 [INSPIRE].

[59] G. Obied, H. Ooguri, L. Spodyneiko and C. Vafa, de Sitter Space and the Swampland, arXiv: 1806.08362 [INSPIRE].

[60] S.K. Garg and C. Krishnan, Bounds on Slow Roll and the de Sitter Swampland, JHEP 11 (2019) 075 [arXiv: 1807.05193] [inSPIRE].

[61] H. Ooguri, E. Palti, G. Shiu and C. Vafa, Distance and de Sitter Conjectures on the Swampland, Phys. Lett. B 788 (2019) 180 [arXiv:1810.05506] [INSPIRE].

[62] V.S. Kaplunovsky and J. Louis, Model independent analysis of soft terms in effective supergravity and in string theory, Phys. Lett. B 306 (1993) 269 [hep-th/9303040] [INSPIRE].

[63] A. Brignole, L.E. Ibáñez and C. Muñoz, Towards a theory of soft terms for the supersymmetric Standard Model, Nucl. Phys. B 422 (1994) 125 [Erratum ibid. 436 (1995) 747] [hep-ph/9308271] [INSPIRE].

[64] A. Brignole, L.E. Ibáñez and C. Muñoz, Soft supersymmetry breaking terms from supergravity and superstring models, Adv. Ser. Direct. High Energy Phys. 18 (1998) 125 [hep-ph/9707209] [INSPIRE].

[65] S. Ferrara, C. Kounnas and F. Zwirner, Mass formulae and natural hierarchy in string effective supergravities, Nucl. Phys. B 429 (1994) 589 [Erratum ibid. 433 (1995) 255] [hep-th/9405188] [INSPIRE].

[66] E. Dudas and S.K. Vempati, Large D-terms, hierarchical soft spectra and moduli stabilisation, Nucl. Phys. B 727 (2005) 139 [hep-th/0506172] [INSPIRE].

[67] L. Randall and R. Sundrum, Out of this world supersymmetry breaking, Nucl. Phys. B 557 (1999) 79 [hep-th/9810155] [INSPIRE].

[68] G.F. Giudice, M.A. Luty, H. Murayama and R. Rattazzi, Gaugino mass without singlets, JHEP 12 (1998) 027 [hep-ph/9810442] [INSPIRE].

[69] J.A. Bagger, T. Moroi and E. Poppitz, Anomaly mediation in supergravity theories, JHEP 04 (2000) 009 [hep-th/9911029] [INSPIRE].

[70] P. Binetruy, M.K. Gaillard and B.D. Nelson, One loop soft supersymmetry breaking terms in superstring effective theories, Nucl. Phys. B 604 (2001) 32 [hep-ph/0011081] [INSPIRE].

[71] K. Choi, A. Falkowski, H.P. Nilles and M. Olechowski, Soft supersymmetry breaking in KKLT flux compactification, Nucl. Phys. B 718 (2005) 113 [hep-th/0503216] [INSPIRE]. 
[72] K. Choi and H.P. Nilles, The Gaugino code, JHEP 04 (2007) 006 [hep-ph/0702146] [INSPIRE].

[73] M. Berg, D. Marsh, L. McAllister and E. Pajer, Sequestering in String Compactifications, JHEP 06 (2011) 134 [arXiv:1012.1858] [INSPIRE].

[74] M. Berg, J.P. Conlon, D. Marsh and L.T. Witkowski, Superpotential de-sequestering in string models, JHEP 02 (2013) 018 [arXiv: 1207.1103] [INSPIRE].

[75] M. Graña, T.W. Grimm, H. Jockers and J. Louis, Soft supersymmetry breaking in Calabi-Yau orientifolds with D-branes and fluxes, Nucl. Phys. B 690 (2004) 21 [hep-th/0312232] [INSPIRE].

[76] T.W. Grimm and J. Louis, The effective action of $N=1$ Calabi-Yau orientifolds, Nucl. Phys. B 699 (2004) 387 [hep-th/0403067] [INSPIRE].

[77] P.G. Cámara, L.E. Ibáñez and A.M. Uranga, Flux induced SUSY breaking soft terms, Nucl. Phys. B 689 (2004) 195 [hep-th/0311241] [INSPIRE].

[78] N. Arkani-Hamed, L. Motl, A. Nicolis and C. Vafa, The String landscape, black holes and gravity as the weakest force, JHEP 06 (2007) 060 [hep-th/0601001] [INSPIRE].

[79] H. Ooguri and C. Vafa, Non-supersymmetric AdS and the Swampland, Adv. Theor. Math. Phys. 21 (2017) 1787 [arXiv:1610.01533] [INSPIRE].

[80] T.D. Brennan, F. Carta and C. Vafa, The String Landscape, the Swampland, and the Missing Corner, PoS TASI2017 (2017) 015 [arXiv: 1711.00864] [inSPIRE].

[81] H.-C. Kim, G. Shiu and C. Vafa, Branes and the Swampland, Phys. Rev. D 100 (2019) 066006 [arXiv: 1905.08261] [INSPIRE].

[82] E. Palti, The Swampland: Introduction and Review, Fortsch. Phys. 67 (2019) 1900037 [arXiv: 1903.06239] [INSPIRE].

[83] L. Randall, The Boundaries of KKLT, Fortsch. Phys. 68 (2020) 1900105 [arXiv: 1912.06693] [INSPIRE]. 